\title{
Comparison of Pocket Pulse Oximeter and Standard Pulse Oximeter With ABG Analysis in Critically III Patients
}

\author{
Jeswanth Reddy N. ${ }^{1}$, Kiran H. S. ${ }^{2^{*}}$, B. J. Subhash Chandra ${ }^{2}$, Basavana \\ Gowdappa $H_{0}^{2}$
}

${ }^{1}$ Department of General Medicine, Melmaruvathur Adhiparasakthi Institute of Medical Sciences \& Research, Melmaruvathur, Kancheepuram district, Tamil Nadu, India

${ }^{2}$ Department of General Medicine, JSS Medical College and Hospital, JSSAHER, Mysore, Karnataka, India

ORCID:

Kiran H. S.: https://orcid.org/0000-0002-6070-6529

Corresponding Author: Kiran H. S.; email:

drhskiran@gmail.com

Received 12 October 2021

Accepted 01 December 2021

Published 31 December 2021

Production and Hosting by Knowledge E

(c) Jeswanth Reddy N. et al.. This article is distributed under the terms of the

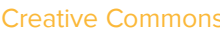

Attribution License, which permits unrestricted use and redistribution provided that the original author and source are credited.

Editor-in-Chief:

Prof. Mohammad A. M. Ibnouf

\section{Abstract}

Background: Pulse oximetry $\left(\mathrm{SpO}_{2}\right)$ is a standard monitoring device in patients presenting to EMDs and intensive care units (ICUs). Pocket pulse oximeters (PPOs) are used widely in wards, EMDs, and small hospitals/clinics. These inexpensive PPOs also guide therapeutic interventions. Few studies have evaluated the accuracy of $\mathrm{SpO}_{2}$ in patients presenting to critical care areas vis-à-vis devices like PPO and standard pulse oximeter (SPO). This study becomes extremely relevant in view of the ongoing crisis of the COVID-19 pandemic wherein $\mathrm{SpO}_{2}$ monitoring is very important in hospitals, quarantine centers, small clinics, or even at home.

Methods: Patients presenting to critical areas who underwent arterial blood gases (ABG) analysis on the recommendation of the treating physician between November 2016 and October 2018 were included in this study. Along with the ABG analysis, a simultaneous assessment of $\mathrm{SpO}_{2}$ was done with a single PPO and SPO and all values were noted. Statistical analysis was done using the SPSS v.21.0 for Windows.

Results: The study included 300 patients. We compared the $\mathrm{O}_{2}$ saturations of ABG, SPO, and PPO with respect to sex, different age groups, and at different levels of $A B G$ $\mathrm{pCO}_{2}, \mathrm{HCO}_{3}$, and $\mathrm{pH}$ in all patients. All parameters were compared using the Pearson's correlation test; the results showed that $A B G \mathrm{O}_{2}$ saturations were closer to the SPO than the PPO but the differences were not statistically significant as the Pearson's correlation values for all parameters were $>0.8$. We also compared the parameters by Bland Altman Plot and all observations were outside $95 \% \mathrm{Cl}$ (confidence interval), which means that there was a good agreement between $\mathrm{O}_{2}$ saturations by all three methods, that is, $A B G, S P O$, and PPO; however, $A B G \mathrm{O}_{2}$ saturations were closer to SPO than PPO but this difference was not statistically significant. Hence, we conclude that the PPO is a useful tool for reliable monitoring of $\mathrm{O}_{2}$ saturations.

Conclusion: This study highlights that inexpensive and noninvasive PPO can be used as a standard monitoring device with reliability in critically ill patients presenting to EMDs, ICUs, and small hospitals/clinics, quarantine centers, and even at home. 


\section{Introduction}

Pulse oximeter is a device for noninvasive measurement of $\mathrm{SpO}_{2}$ (oxygen saturation) in blood [1].Sending specific wavelengths of light through nail bed based on differential absorption of the wavelengths of the light by deoxygenated and oxygenated hemoglobin, the pulse oximeter calculates and shows an $\mathrm{SpO}_{2}$ value [1]. Nowadays, pulse oximeter $\mathrm{SpO}_{2}$ is considered as a "fifth vital sign" (in addition to temperature, blood pressure, pulse, and respiratory rate) in clinical assessment [2].Ever since its inception in 1970s, it is being used in various clinical settings [3].Pocket pulse oximeters (PPOs) are increasingly being used in wards, critical care areas, peripheral clinics, etc. Their small size, handy nature, and affordable cost have established their role in modern clinical care just like thermometers in the management of patients with fever [3]. However, these commercially available pocket devices have not been adequately evaluated [2]. Hence, we wanted to find out the reliability of pocket pulse oximeter (PPO) vis-à-vis standard pulse oximeter (SPO - which comes with a finger probe connected to a standard monitor), and ABG (arterial blood gases) analysis. Although this study was done in pre-COVID era, it becomes extremely relevant in view of the ongoing crisis of COVID-19 pandemic wherein $\mathrm{SpO}_{2}$ monitoring is very important in hospitals, quarantine centers, small clinics, or even at home [4, 5].

\section{Materials and Methods}

This observational prospective and comparative study was undertaken at a medical college teaching hospital. The study included 300 patients admitted to critical care areas between November 2016 and October 2018. Consecutive patients presented to critical areas who underwent $A B G$ analysis as per the decision of the treating physician were included by convenience sampling. Patients with shock in whom pulse oximetry does not record the saturation $\left(\mathrm{SpO}_{2}\right)$ and patients with anemia and abnormal hemoglobin were excluded. Along with the ABG analysis, a simultaneous assessment of $\mathrm{SpO}_{2}$ was done with a single PPO and SPO, and all values were noted. $\mathrm{SpO}_{2}$ obtained by PPO, SPO were compared with each other and with various parameters of $A B G$ analysis. To ensure the accuracy of the measurement, the values were recorded only after the PPO sensor showed a steady pulse and/or pulse waveform. 
TABLE 1: Descriptive statistics comparing SPO vs $A B G \mathrm{O}_{2}$ saturation.

\begin{tabular}{|l|l|l|l|l|l|} 
& N & Minimum & Maximum & Mean & $\begin{array}{l}\text { Std. } \\
\text { Deviation }\end{array}$ \\
\hline $\begin{array}{l}\text { Difference between } \\
\text { and } \mathrm{SPO} \mathrm{SPO}_{2} \%\end{array}$ & $\mathrm{ABG} 300$ & -10.00 & 5.9 & -0.2262 & 1.65147 \\
\hline
\end{tabular}

TABLE 2: Descriptive statistics comparing PPO vs $A B G \mathrm{O}_{2}$ saturation.

\begin{tabular}{|l|l|l|l|l|l|} 
& \multicolumn{1}{|c}{$\mathbf{N}$} & Minimum & Maximum & Mean & $\begin{array}{l}\text { Std. } \\
\text { Deviation }\end{array}$ \\
$\begin{array}{llllll}\text { Difference between } \\
\text { and } \mathrm{PPO} \mathrm{SPO}_{2} \%\end{array}$ & $\mathrm{ABG} 300$ & -13 & 6.9 & 0.46 & 1.727 \\
\hline
\end{tabular}

\section{Results}

Out of the 300 patients included in the study, $69 \%$ were male and $31 \%$ female. Pearson's correlation showed that $\mathrm{ABG} \mathrm{O}_{2}$ saturation was closer to SPO than PPO in both males and females; however, these differences were within the acceptable limits. Hence, there was no statistically significant difference between males and females for monitoring $\mathrm{O}_{2}$ saturation by PPO.

Furthermore, $5 \%$ of the patients were $<20$ years old, $27 \%$ between 20 and $40,34 \%$ between 40 and 60, 29\% between 60 and 80, and 5\% >80. Across all age groups, $A B G O_{2}$ saturations were closer to SPO than PPO but this difference was not significant as the Pearson's correlation values were $>0.8$, which is considered to have excellent correlation. Hence, age does not have any statistically significant influence on PPO saturation monitoring.

According to the Pearson's correlation, there was an excellent correlation between the saturation recorded by PPO and SPO with $A B G O_{2}$ saturation. SPO values were closer to $A B G \mathrm{O}_{2}$ saturation than PPO but this difference was not significant.

Linear regression showed standardized coefficient error of 0.868 and 0.878 with PPO and SPO, respectively, when compared with the saturations of ABG which is insignificant as the standardized coefficient error is $<0.1$ (standardized coefficient error $<0.1$ is insignificant).

Only 10/300 (3.3\%) patients were beyond 95\% Cl (Confidence interval) which means there is a good agreement between the two methods ( $A B G \mathrm{SaO}_{2}$ and SPO).

Overall, $13 / 300$ (4.3\%) observations were outside $95 \% \mathrm{Cl}$, which means there is still a good agreement between the two methods ( $A B G \mathrm{SaO}_{2}$ and PPO).

Only $6 / 300$ (2\%) had $>95 \% \mathrm{Cl}$, which means there is a good agreement between the two methods. 
Bland Altman Plot of comparing Standard Pulse Oximeter vs ABG $\mathrm{O}_{2}$ saturation

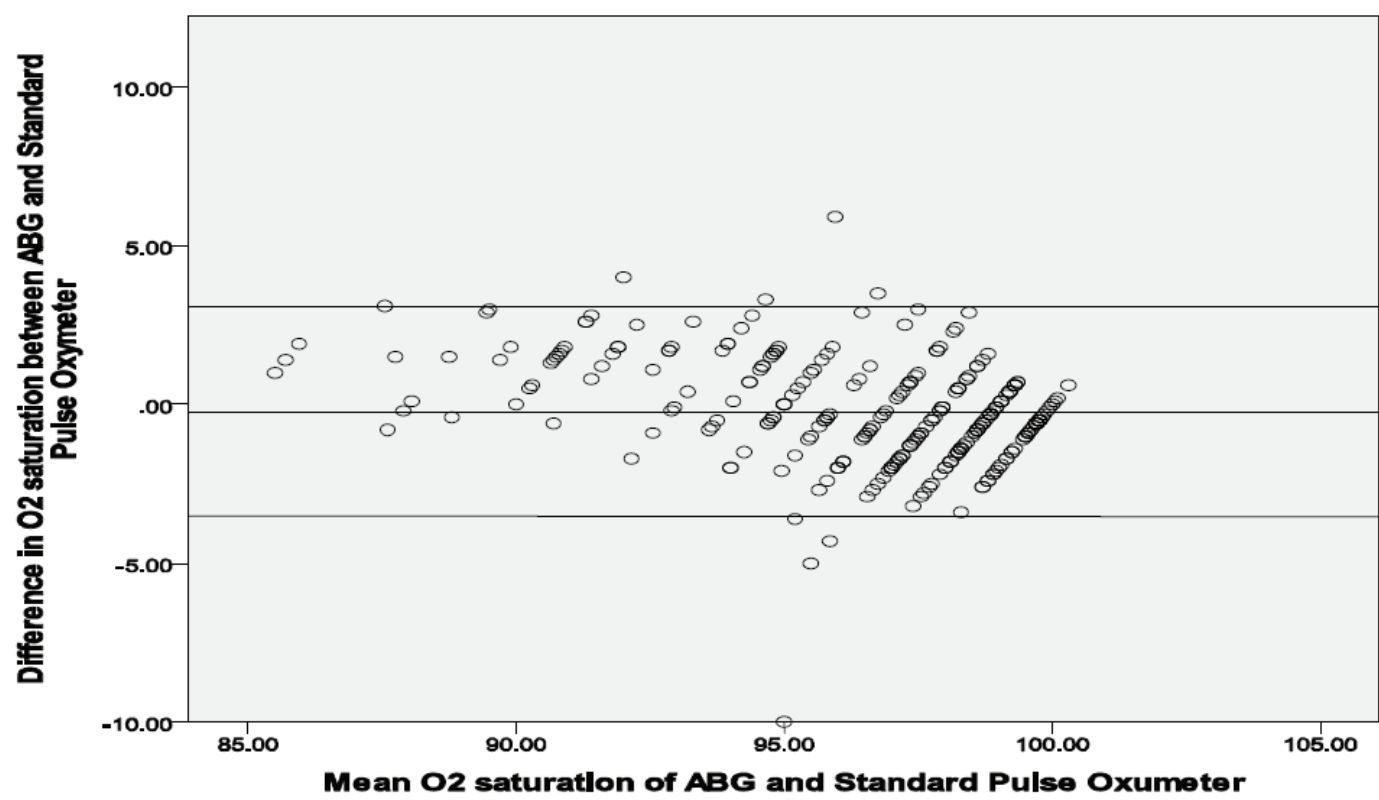

Figure 1: Bland Altman Plot comparing SPO vs $A B G O_{2}$ saturation.

\section{Bland Altman Plot of comparing Pocket Pulse Oximeter vs $\mathrm{ABG} \mathrm{O}_{2}$ saturation}

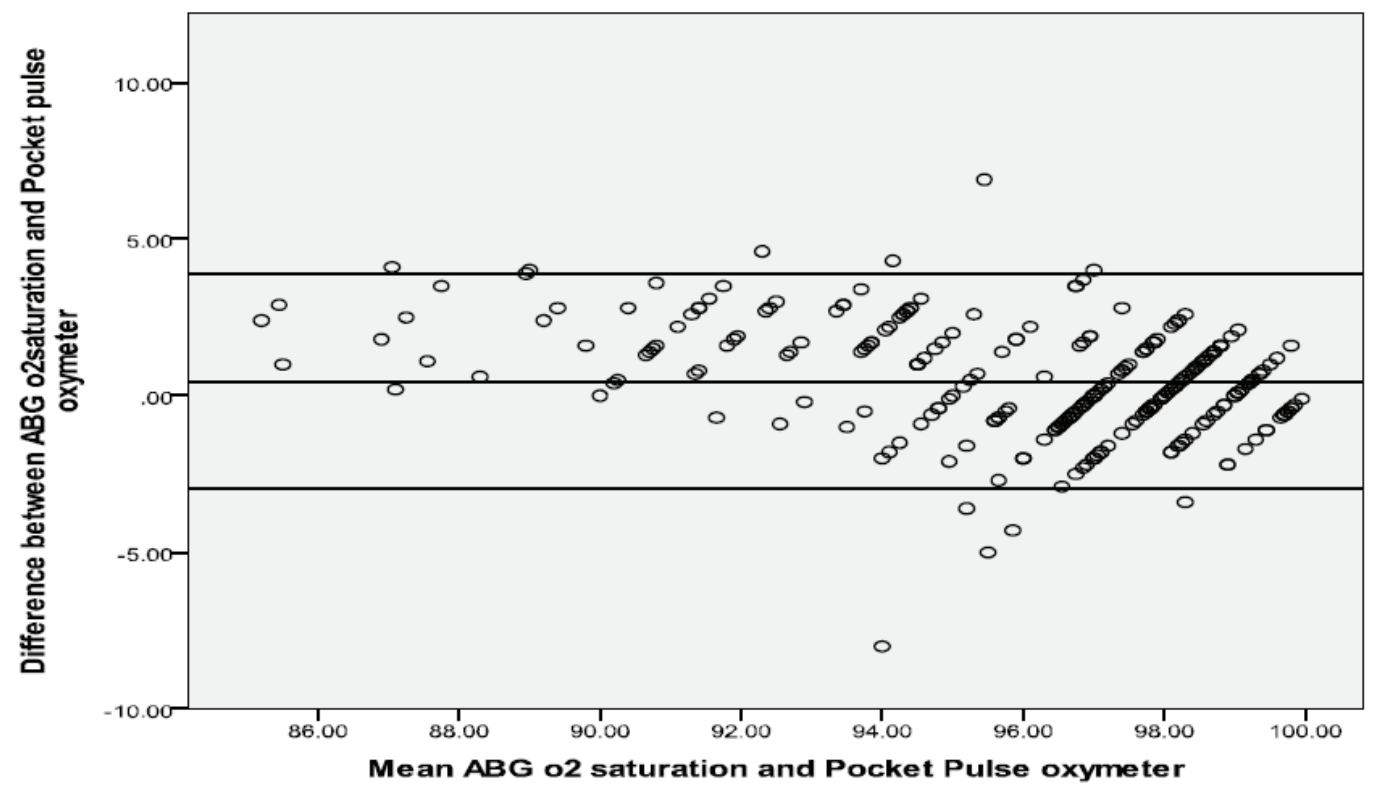

Figure 2: Bland Altman Plot comparing PPO vs $A B G \mathrm{O}_{2}$ saturation.

Finally, to conclude, based on the Bland Altman Plot, while ABG is in better agreement with SPO than PPO, both were within the acceptable limits. 


\section{Bland Altman Plot of Agreement between PPO vs SPO}

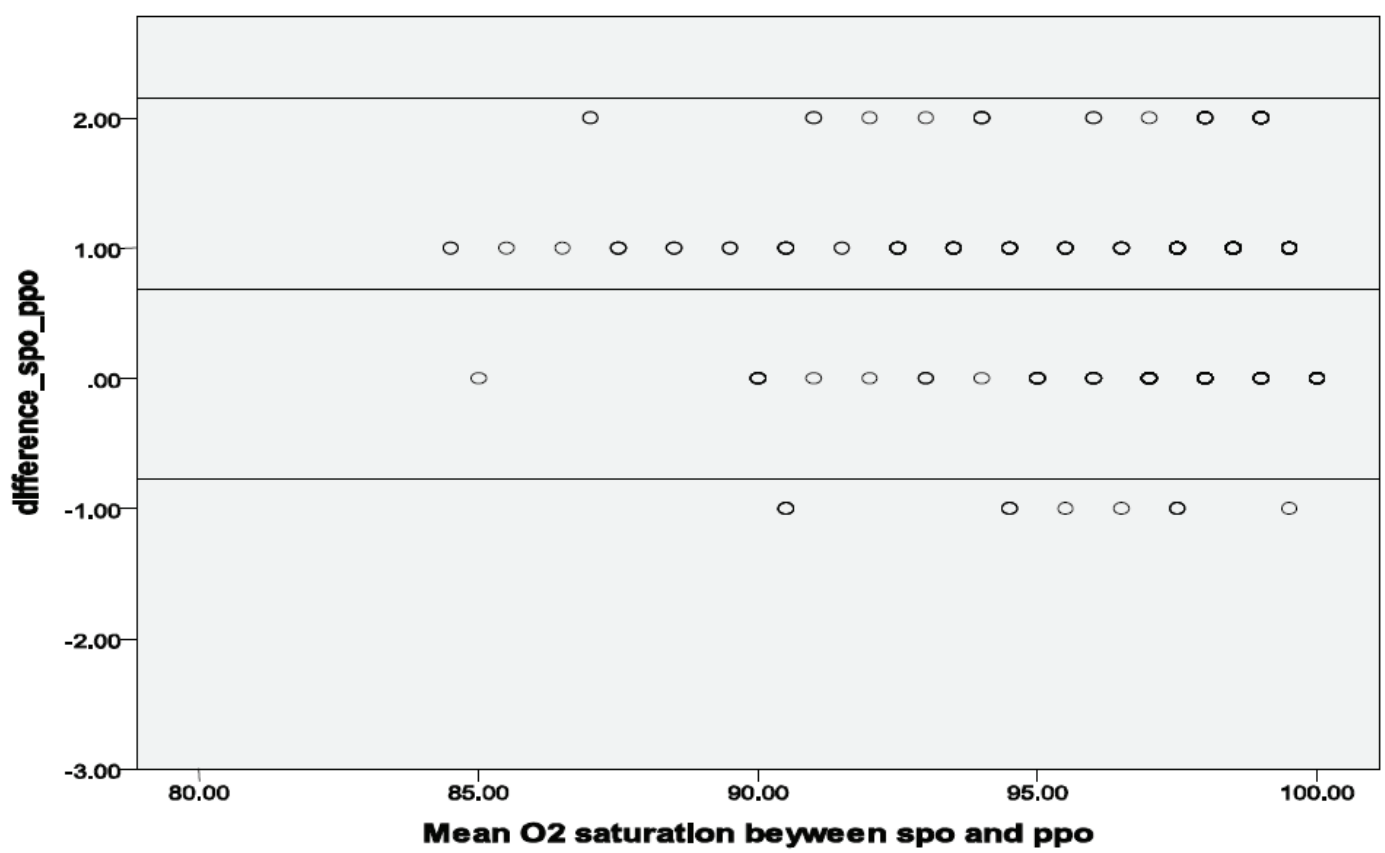

Figure 3: Bland Altman Plot of agreement between PPO vs SPO.

TABLE 3: Descriptive statistics showing agreement between PPO and $A B G \mathrm{O}_{2}$ saturation.

\begin{tabular}{l|l|l|l|l|l|l|l|} 
& Mean & Median & $\begin{array}{l}\text { Standard } \\
\text { deviation }\end{array}$ & Minimum & Maximum & $\begin{array}{l}\text { Percentile } \\
\mathbf{2 5}\end{array}$ & $\begin{array}{l}\text { Percentile } \\
\mathbf{7 5}\end{array}$ \\
$\begin{array}{l}\text { Difference } \\
\text { between SPO }\end{array}$ & 0.69 & 1.00 & 0.73 & -1 & 2.00 & 0.00 & 1.00 \\
and PPO
\end{tabular}

Hence, based on the Bland Altman Plot, PPO can be a reliable tool for monitoring saturation. We also compared the saturations of all three, that is, ABG, PPO, and $\mathrm{SPO}$ at different levels of $\mathrm{ABG} \mathrm{PCO}_{2}$ values to determine whether $\mathrm{PCO}_{2}$ levels have any influence. Results showed that there was a better correlation between ABG and SPO $\mathrm{O}_{2}$ saturations than PPO but the difference was within the acceptable limit. So, $\mathrm{ABG} \mathrm{PCO}_{2}$ does not influence the saturation monitoring by PPO. We also compared the saturations of all three, that is, ABG, PPO, and SPO at different levels of $\mathrm{ABG} \mathrm{HCO}_{3}$ values to determine whether $\mathrm{HCO}_{3}$ level has any influence. Results showed that there was a better correlation between ABG and SPO than between $A B G$ and PPO but the difference was within the acceptable limit. So, $\mathrm{HCO}_{3}$ level does not influence the saturation monitoring by PPO. In addition, neither respiratory acidosis/alkalosis nor metabolic acidosis/alkalosis affect the PPO saturation monitoring. Both in metabolic acidosis and alkalosis $A B G$, values of $\mathrm{O}_{2}$ saturation are closer 
to SPO than PPO but within acceptable limits. Similarly, in respiratory acidosis or alkalosis, $\mathrm{ABG}$ values of $\mathrm{O}_{2}$ saturation are closer to SPO than PPO but within acceptable limits.

\section{Discussion}

PPO is nowadays a ubiquitous, disseminated technology used for monitoring $\mathrm{O}_{2}$ saturation in patients presenting to critical care units such as EMDs, ICUs, small hospitals/clinics owing to their ease of use and the ability to provide continuous and immediate oxygen saturation values [1-3]. Furthermore, PPO is a noninvasive method for monitoring oxygen saturation $\left(\mathrm{SO}_{2}\right)$, so knowing its precision and accuracy is of great importance in clinical practice [1, 2]. Hence, this study was carried out to compare the $\mathrm{O}_{2}$ saturation of PPO with that of SPO and $A B G \mathrm{O}_{2}$ saturations and to examine the effect of several factors on this relationship. Our study showed that $\mathrm{SPO}_{2}$ saturation was more closely related to $A B G$ values than PPO across all parameters, that is, sex, different age groups, and at different levels of $A B G \mathrm{pCO}_{2}, \mathrm{HCO}_{3}$, and $\mathrm{pH}$. Similarly, $\mathrm{ABG} \mathrm{O}_{2}$ saturation was more closely related to SPO than PPO across all parameters. There was a better correlation between ABG and SPO than PPO, however, as per the statistical analysis, those differences were within acceptable limits and statistically insignificant. Hence, inexpensive PPO can be used as a standard monitoring device with reliability in critically ill patients.

Van de Louw A et al. noted large differences between saturation measured by PPO and $A B G$ in critically ill patients with poor reproducibility of $\mathrm{SpO}_{2}$ [6], however, our study showed that there was an excellent correlation between the saturations recorded by PPO, SPO, and ABG (as the Pearson's correlation values $>0.8$ are considered to have excellent correlation; linear regression showed a standardized coefficient error of 0.868 which occurred between PPO and ABG saturation which is insignificant as the standardized coefficient error is $<0.1$ which is considered insignificant; Bland Altman Plot also showed an excellent correlation between the two as 13/300 (4.3\%) observations were outside $95 \% \mathrm{Cl}$, which means that there is a good agreement between the two methods).

Da Costa JC et al. showed that PPO was less precise than SPO but had agreement limits that were comparable [7]. Our study also showed the same results. Moreover, Van de Louw et al. [6] compared only ABG and PPO and da Costa JC et al. [7] compared only between SPO and PPO, but in this study, we compared all three methods of monitoring 
$\mathrm{O}_{2}$ saturation, that is, $\mathrm{ABG}, \mathrm{SPO}$, and PPO, which showed a good correlation between all three methods.

Wilson et al. showed that PPO overestimates ABG-determined $\mathrm{SaO}_{2}$ by a mean of $2.75 \%$ in patients with sepsis and septic shock [8]. This overestimation is exacerbated by the presence of hypoxemia. When $\mathrm{SaO}_{2}$ needs to be determined with a high degree of accuracy, ABG is recommended. In our study, we have excluded patients with shock, hence the issue does not arise at all.

Moreover, Seguin et al. demonstrated that neither anemia nor acidosis alters the precision of measurements, thus PPO remains a valuable tool in the care of ICU patients [9]. In this study, we excluded patients with anemia. In our study also, acidosis, either respiratory or metabolic, does not alter the precision of measurement as the Pearson's correlation values were $>0.8$, which means that there exists a good correlation. In our study, we also compared $\mathrm{O}_{2}$ saturations at different levels of $A B G$ alkalosis, that is, both respiratory and metabolic alkalosis and results showed that alkalosis also do not alter the precision of measurement as the Pearson's correlation values obtained were $>0.8$.

Munoz et al. reported that carbon dioxide arterial tension status can impair the agreement between arterial oxygen saturation and arterial oxygen saturation measured by pulse oximetry, particularly in patients with hypercapnia [10]. Therefore, it is likely that arterial oxygen saturation measured by oximetry may not be sufficiently accurate when assessing patients for long-term home oxygen therapy and should not substitute the gold standard arterial oxygen tension measured in arterial blood. Our study also showed that there exists differences between the saturations recorded by PPO and $A B G$ at different levels of $A B G p \mathrm{pO}_{2}$, but these differences were within agreeable limits by Pearson's correlation.

One of the most important part of COVID-19 patient management is PPO more so because of "silent hypoxia" [3, 11, 12]. Many countries have adopted PPO at primary healthcare/home settings [3].In a recent study done to assess the correlation between PPO $\left(\mathrm{SpO}_{2}\right)$ and $\mathrm{ABG}$ analysis $\left(\mathrm{SaO}_{2}\right)$ in patients with COVID-19, who were fit to be shifted from an ICU to a ward, it was found that PPO correlated with $\mathrm{SaO}_{2}$ measurement; although suboptimal, it was within the acceptable levels for Food and Drug Authority approval [13] 


\section{Limitations and Highlights}

In this study, we excluded patients with shock, anemia, and abnormal hemoglobin levels [14]. Hence, we could not estimate the correlation between ABG, PPO, and SPO in the above situations. This is the first ever study of its kind in our country since we compared, simultaneously, saturations recorded by PPO and SPO with ABG which, to the best of our knowledge, none of the other studies have done so far.

\section{Conclusion}

This study highlights that inexpensive PPO can be used as a standard monitoring device with reliability in critically ill patients presenting to EMDs, ICUs, and small hospitals/clinics in the periphery; more so, during the current COVID-19 pandemic.

\section{Acknowledgements}

The authors dedicate this article to Dr Takuo Aoyagi (who passed away last year) - "the father of the pulse oximeter" - for his contributions in its development [15]. The authors are also thankful to their laboratory department for its support.

\section{Ethical Considerations}

Institutional Ethics Committee approval and informed consent of patients were obtained.

\section{Competing Interests}

None.

\section{Availability of Data and Material}

All relevant data and methodological details pertaining to this study are available to any interested researchers upon reasonable request to the corresponding author. 


\section{Funding}

None.

\section{References}

[1] Torp, K. D., Modi, P., and Simon, L. V. (2021). Pulse oximetry. Treasure Island, FL: StatPearls Publishing.

[2] Hafen, B. B. and Sharma, S. (2021). Oxygen saturation. Treasure Island, FL: StatPearls Publishing.

[3] Singh, A., Kataria, S., Das, P., et al. (2020). A proposal to make the pulse oximetry as omnipresent as thermometry in public health care systems. Journal of Global Health, vol. 10, no. 2, p. 0203102.

[4] Michard, F., Shelley, K., and L'Her, E. (2021). COVID-19: pulse oximeters in the spotlight. Journal of Clinical Monitoring and Computing, vol. 35, no. 1, pp. 11-14.

[5] Quaresima, V. and Ferrari, M. (2020). More on pulse oximetry for monitoring patients with COVID-19 at home. Annals of the American Thoracic Society, vol. 17, no. 11, p. 1496.

[6] Van de Louw, A., Cracco, C., Cerf, C., et al. (2001). Accuracy of pulse oximetry in the intensive care unit. Intensive Care Medicine, vol. 27, no. 10, pp. 1606-1613.

[7] da Costa, J. C., Faustino, P., Lima, R., et al. (2016). Comparison of the accuracy of a pocket versus standard pulse oximeter. Biomedical Instrumentation \& Technology, vol. 50, no. 3, pp. 190-193.

[8] Wilson, B. J., Cowan, H. J., Lord, J. A., et al. (2010). The accuracy of pulse oximetry in emergency department patients with severe sepsis and septic shock: a retrospective cohort study. BMC Emergency Medicine, vol. 10, no. 9.

[9] Seguin, P., Le Rouzo, A., Tanguy, M., et al. (2000). Evidence for the need of bedside accuracy of pulse oximetry in an intensive care unit. Critical Care Medicine, vol. 28, no. 3, pp. 703-706.

[10] Muñoz, X., Torres, F., Sampol, G., et al. (2008). Accuracy and reliability of pulse oximetry at different arterial carbon dioxide pressure levels. European Respiratory Journal, vol. 32, no. 4, pp. 1053-1059.

[11] Wilkerson, R. G., Adler, J. D., Shah, N. G., et al. (2020). Silent hypoxia: a harbinger of clinical deterioration in patients with COVID-19. American Journal of Emergency Medicine, vol. 38, no. 10, pp. 2243.e5-2243.e6. 
[12] Brouqui, P., Amrane, S., Million, M., et al. (2021). Asymptomatic hypoxia in COVID-19 is associated with poor outcome. International Journal of Infectious Diseases, vol. 102, pp. 233-238.

[13] Philip, K. E. J., Bennett, B., Fuller, S., et al. (2020). Working accuracy of pulse oximetry in COVID-19 patients stepping down from intensive care: a clinical evaluation. BMJ Open Respiratory Research, vol. 7, no. 1, p. e000778.

[14] Moyle, J. (2021). Pulse oximetry gives artificially high readings in presence of carboxyhaemoglobin. BMJ, vol. 373, p. n1103.

[15] Yamakage, M. (2021). Pulse oximetry: the outstanding achievements of Dr. Takuo Aoyagi. Journal of Anesthesia, vol. 35, article 605. 